\title{
Neumotórax espontáneo bilateral metastásico. Inusual manifestación de metástasis pulmonar
}

\author{
Jorge Lavanderos F..$^{1,2}$, Jaime Jans B. ${ }^{1,2}$, Marcelo Devaud J. ${ }^{1,2}$ y Hellmar Konrad F. ${ }^{3}$
}

\section{Spontaneous metastatic bilateral pneumothorax. Unusual manifestation of lung metastasis}

\begin{abstract}
Aim: To report the case of a 77-year-old patient treated for angiosarcoma of the scalp, who evolves with bilateral pneumothorax, whose histopathological study reports metastatic disease. Materials and Method: Review of electronic clinical record, computerized imaging record and biopsy reports. Outpatient followup at the polyclinic of surgery. Results: Management was performed by bilateral videothoracoscopy, with bulectomy plus pleurodesis. Discussion: Secondary pneumothorax due to metastatic disease is rare, approximately 1 to $2 \%$ of cases. Sarcomas are among the tumors that most frequently metastasize to the lung. Conclusion: Metastatic pneumothorax is infrequent and requires a high index of suspicion. Its management will depend on the prognosis and the general conditions of the patient.

Key words: pneumothorax; metástasis; angiosarcoma; pleurodesis.
\end{abstract}

\section{Resumen}

Objetivo: Comunicar el caso de una paciente de 77 años tratada de un angiosarcoma de cuero cabelludo, que evoluciona con neumotórax bilateral, cuyo estudio histopatológico informa enfermedad metastásica. Materiales y Método: Revisión de ficha clínica electrónica, registro informático de imagenología e informes de biopsias. Seguimiento ambulatorio en policlínico de cirugía. Resultados: Se realiza manejo por videotoracoscopía bilateral, con bulectomía más pleurodesis. Discusión: El neumotórax secundario por enfermedad metastásica es poco frecuente, aproximadamente $1 \%$ a $2 \%$ de los casos. De los tumores que más frecuentemente metastizan al pulmón se encuentran los sarcomas. Conclusión: El neumotórax metastásico es poco frecuente y requiere un alto índice de sospecha. Su manejo va a depender del pronóstico y de las condiciones generales del paciente.

Palabras clave: neumotórax; metástasis; angiosarcoma; pleurodesis.

\section{Caso clínico}

Paciente de 77 años, con antecedente de reciente tratamiento de tumor de cuero cabelludo (resección oncológica más injerto dermo-epidérmico, más radioterapia adyuvante), cuyo diagnóstico histológico corresponde a un angiosarcoma de alto grado, pT2N0M0. Una vez terminada la radioterapia consulta por dolor torácico y disnea. Estudio imagenológico con radiografía de tórax y luego con tomografía computada de tórax evidencia neumotórax izquierdo de moderada cuantía. Asociado a ello, se evidencian algunas pequeñas lesiones quísticas pulmonares bilaterales que no se encontraban en estudios previos (Figura 1). Se maneja con pleurostomía con evolución favorable. Un mes posterior al episodio consulta nuevamente por dolor torácico y disnea. Evaluación con tomografía computada evidencia hidroneumotórax bilateral, severo a derecha y moderado a izquierda, con importante progresión de las lesiones quísticas del parénquima pulmonar (Figuras 2 y 3 ). Se realiza exploración por videotoracoscopía biportal, bilateral, en dos tiempos. A derecha se encuentran varias bulas pequeñas en lóbulo superior y medio. Se realiza bulectomía con endograpadora de todas las lesiones, más pleurodesis mécanica y química con talco pulverizado. A izquierda se encuentran
Servicio de Cirugía, Hospital Base Osorno. Osorno, Chile. ${ }^{2}$ Campo Clínico Osorno, Facultad de Medicina, Universidad Austral de Chile. Osorno, Chile. ${ }^{3}$ Unidad de Anatomía Patológica, Hospital Base Osorno. Osorno, Chile.

Recibido 2020-08-05 y aceptado 2020-09-21

Correspondencia a: Dr. Jorge Lavanderos F. jlavanderosfernandez@yahoo. com 


\section{CASOS CLÍNICOS}

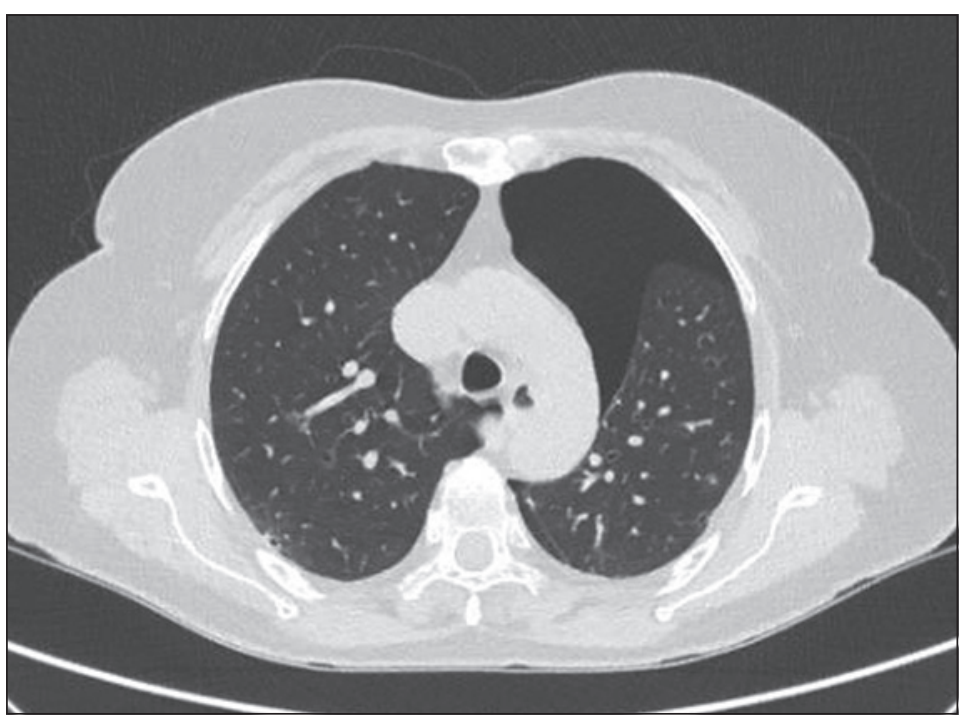

Figura 1. Neumotórax izquierdo primer episodio.

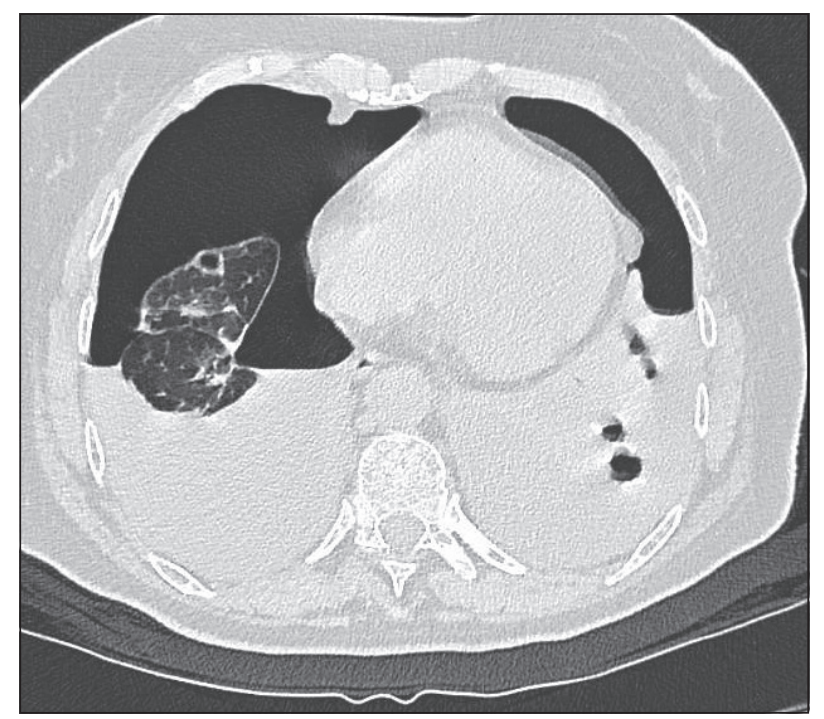

Figura 2. Hidroneumotórax bilateral. pequeñas bulas en lóbulo superior. Se realiza bulectomía de todas las lesiones y pleurodesis de la misma forma que al lado derecho. Evolución

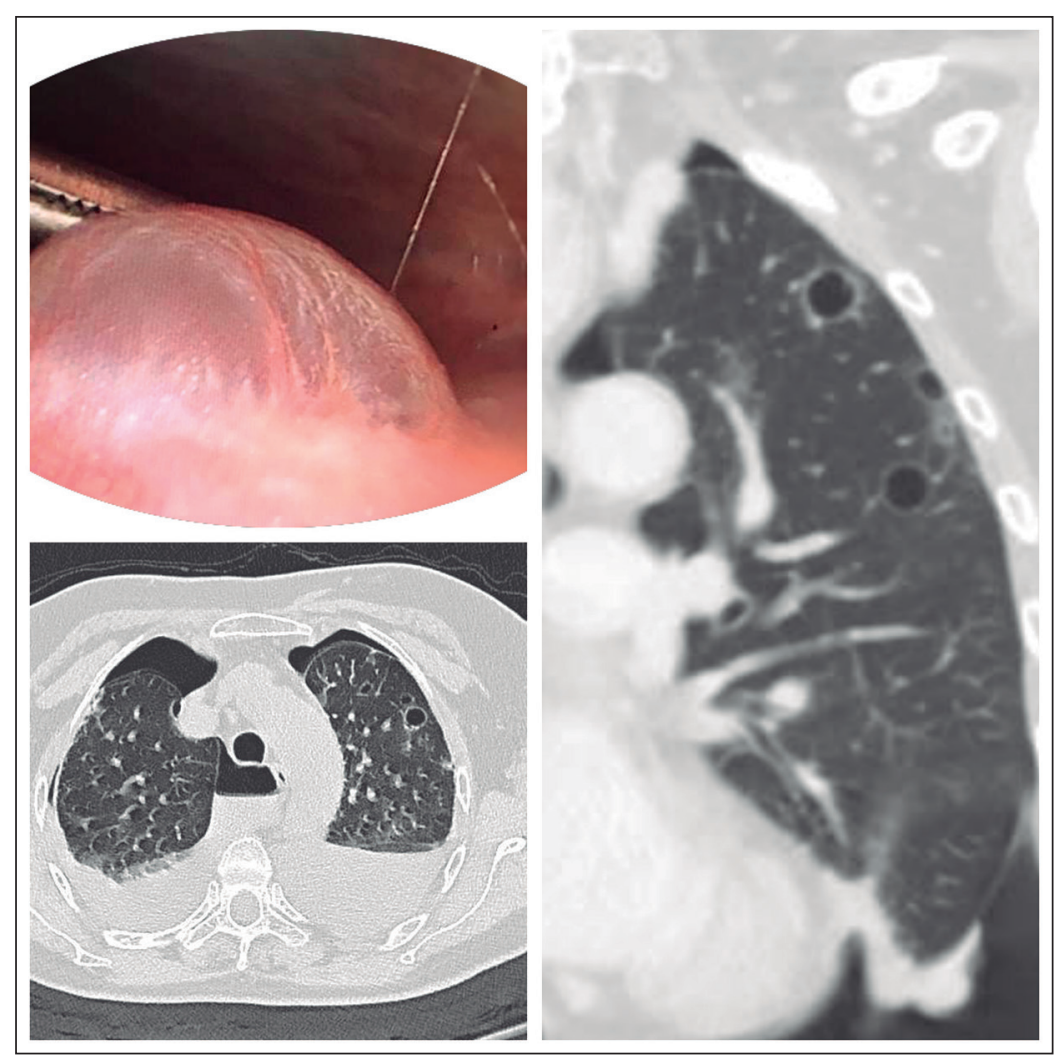

Figura 3. Progresión de la enfermedad quística y bula resecada. favorable, con pulmón expandido y sin derrame en seguimiento en policlínico de cirugía de tórax (Figura 4).

\section{Biopsia}

Signos morfológicos e inmunohistoquímicos compatibles con metástasis pleuropulmonar (bula y pleura parietal) de angiosarcoma. CD31 positivo (Figura 5).

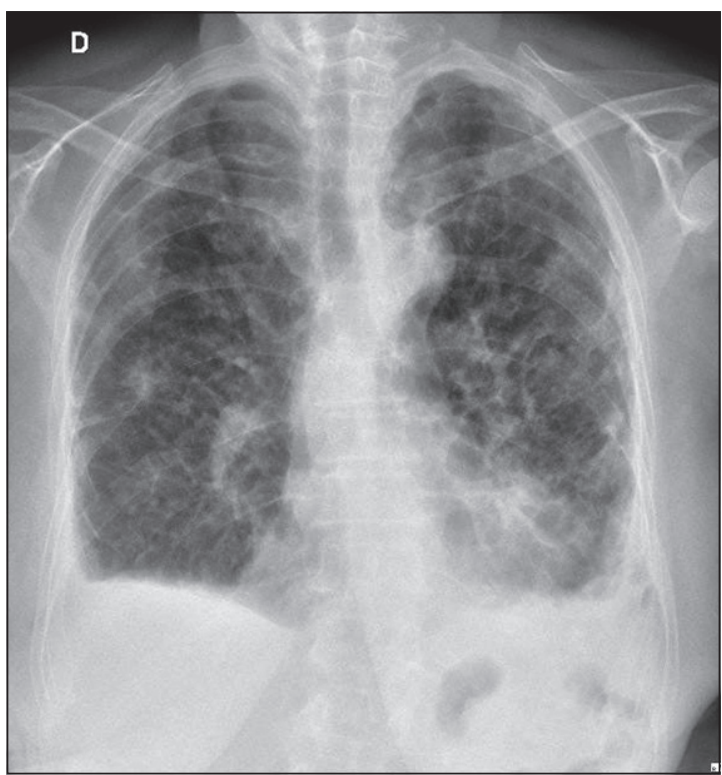

Figura 4. Radiografía de tórax de seguimiento ambulatorio. 


\section{Discusión}

El neumotórax secundario se relaciona fundamentalmente al enfisema buloso, pero hay otras enfermedades del parénquima pulmonar que también se describen en su etiología ${ }^{1}$.

El neumotórax por enfermedad metastásica es poco frecuente, pero debe ser considerado en pacientes con historia de enfermedad neoplásica. Este corresponde al 1\% a 2\% de los neumotórax espontáneos ${ }^{2,3}$.

Se asocia a tumores primarios del pulmón, bulas o nódulos excavados metastásicos, linfangitis carcinomatosa, etc. Los tumores primarios pulmonares son a menudo excavados; por el contrario, sólo el $4 \%$ de los nódulos metastásicos son excavados. Los tumores que más frecuente se ven relacionados son aquellos derivados de los epitelios, tumores genitales y sarcomas².

Existen varias hipótesis para su patogénesis:

- Constitución de una fístula entre el parénquima pulmonar y la pleura, debido a necrosis de un nódulo tumoral subpleural secundario a lesión vascular por quimioterapia o radioterapia ${ }^{2-4}$.

- Obstrucción bronquial parcial por tumor, con efecto de válvula; con distensión y dehiscencia de la pared alveolar formando bulas o neumatoceles que pueden romperse ${ }^{2,4}$.

- Embolia tumoral con infarto y necrosis de la pleura.

- Infiltración tumoral de la pared de una preexistente cavidad que se rompe hacia el espacio pleural ${ }^{2}$.

En la evaluación inicial hasta un 5,5\% de los pacientes no tiene lesiones visibles en la tomografía computada ${ }^{3}$.

Los sarcomas metastizan principalmente al pulmón, hasta el $20 \%$ de los pacientes experimentan esta complicación. En general tienen mal pronóstico; sin embargo, el pronóstico reportado en la literatura varía desde $33 \%$ a $85 \%$ de sobrevida a 5 años, dependiendo del tipo celular de sarcoma analizado ${ }^{1}$.

La recidiva en este grupo de pacientes es algo frecuente, se ha visto que hasta la mitad de ellos presenta más de un episodio de neumotórax ${ }^{1,4}$.

$\mathrm{El}$ angiosarcoma por su parte, es una neoplasia altamente maligna que se origina en el endotelio vascular y se desarrolla principalmente en cuero cabelludo y cara en la población mayor; pueden metastizar a pulmón (principalmente), hígado, linfonodos y piel $^{5}$. Las metástasis pulmonares causan la mayoría de las muertes debido a complicaciones respiratorias ${ }^{6}$.

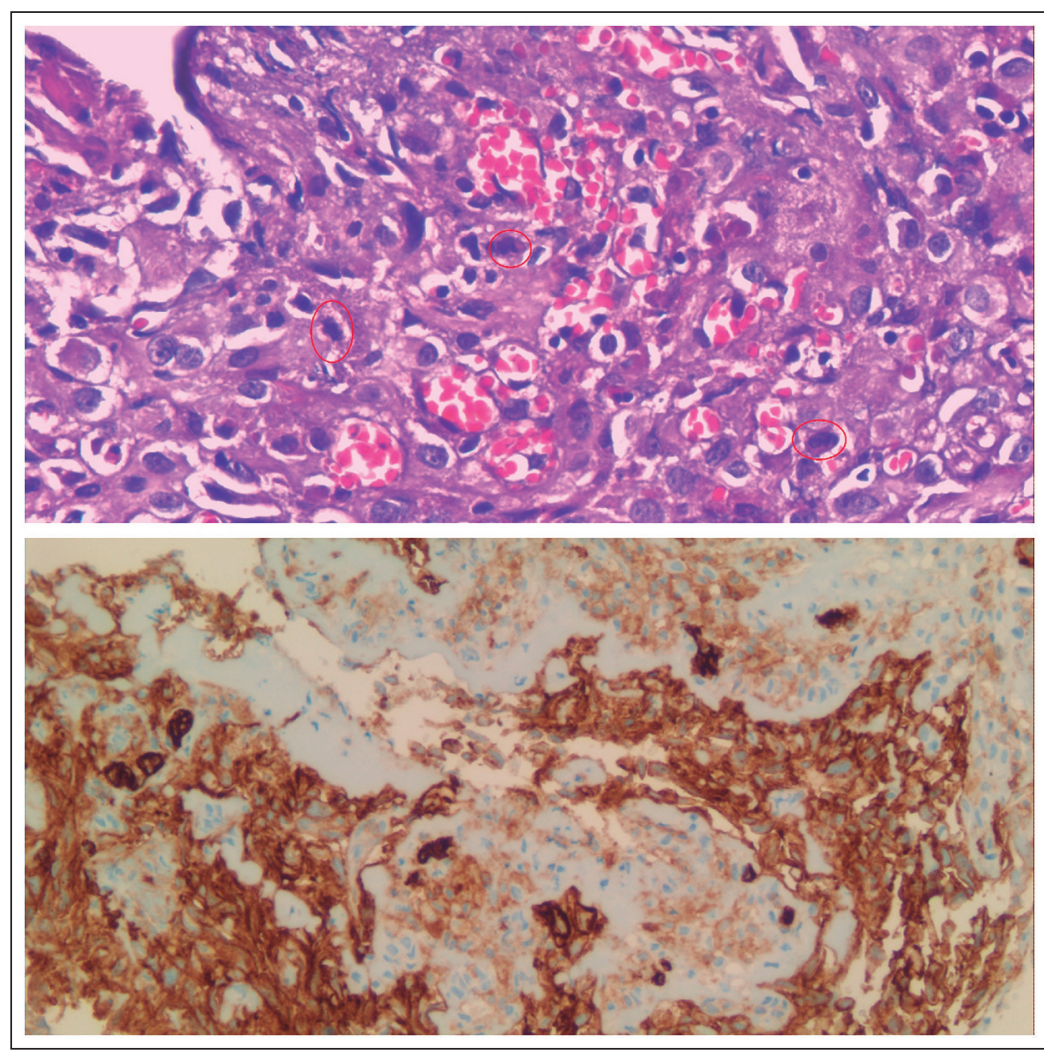

Figura 5. Superior: Tinción H-E. Parénquima pulmonar con pequeños focos de infiltración de una neoplasia que tiene tendencia a formar pequeños vasos. Inferior: Inmunohistoquímica CD31 $(+)$

El síntoma más frecuente de presentación de metástasis pulmonar en angiosarcoma es la hemoptisis, y solo unos pocos casos presentan neumotórax espontáneo o neumomediastino. Las manifestaciones tomográficas más comunes son múltiples lesiones nodulares sólidas o múltiples quistes de pared delgada, a menudo con cambios hemorrágicos ${ }^{7}$.

El PET-TC (glucosa) comúnmente usado en la estadificación de neoplasias, no otorga mayor información que la establecida por la tomografía computada ${ }^{8}$.

En el estudio histopatológico de las lesiones, el análisis inmunohistoquímico se basa en los marcadores de endotelio CD31 y CD34, que suelen dar positivo en los angiosarcomas. El anticuerpo monoclonal D4-40 es un conocido marcador linfático que ha sido reportado como marcador de angiosarcoma, como así también el factor de transcripción ETS (erythroblast transformation-specific) es un sensible marcador de angiosarcoma y otros tumores vasculares ${ }^{6}$.

Los neumotórax pueden llegar a ser una com- 
plicación de las neoplasias quimiosensibles². El inicio de un régimen de quimioterapia basado en doxorrubicina se ha asociado con el desarrollo de neumotórax ${ }^{8}$.

El tratamiento tiene como objetivo paliar los síntomas; en pacientes con mal pronóstico este se limita a un tubo de drenaje torácico. Sin embargo, pacientes en buenas condiciones generales son candidatos a resección de bulas más pleurodesis por técnicas mínimamente invasivas, que tienen la ventaja de la confirmación histopatológica de la enfermedad y otorga la mayor seguridad en términos de disminuir el riesgo de recidiva ${ }^{2,3.8}$.

\section{Conclusión}

El neumotórax secundario a enfermedad metas- tásica es una entidad poco frecuente y requiere un alto índice de sospecha. La cirugía mínimamente invasiva permite realizar un tratamiento seguro en pacientes que se encuentran en buenas condiciones generales, tal como el caso presentado.

\section{Responsabilidades éticas}

Protección de personas y animales. Los autores declaran que para esta investigación no se han realizado experimentos en seres humanos ni en animales.

Confidencialidad de los datos. Los autores declaran que en este artículo no aparecen datos de pacientes.

Conflictos de interés: no hay.

\section{Bibliografía}

1. Hoag J, Sherman M, Fasihuddin Q, Lund MA. Comprehensive review of spontaneous pneumothorax complicating sarcoma. Chest 2010;138:510-8.

2. Le Garff G, Léna H, Corbineau H, Kerbrat $\mathrm{P}$, Delaval $\mathrm{Ph}$. Unusual cause of recurrent pneumothorax: excavated metastasis of Osteosarcoma. Annals Thoracic Surgery 2001;72:2111-3

3. Matsuura $Y$, Ninomiya $H$, Ichinose J, Nakao M, Ishikawa Y, Okumura S. Pathogenesis of secondary spontaneous pneumothorax complicating Osteosarcoma. The Annals of Thoracic Surgery 2020;110:81-3.
4. Nakada T, Okumura S, Kuroda H, Uehara H, Mun M, Sakao Y, et al. Outcome of radical surgery for pulmonary metastatic Osteosarcoma with secondary spontaneous pneumothorax Case Series Report. Ann Thorac Cardiovasc Surg. 2014; Supplement: 574-7.

5. Sakurai H, Hada M, Miyashita Y, Tsukamoto K, Oyama T, Ashizawa I. Simultaneous bilateral spontaneous pneumothorax secondary to metastatic Angiosarcoma of the Scalp: Report of a Case. Surg Today 2006;36:919-22.

6. Masuzawa M, Mikami T, Numata Y, Tokuyama W, Murakumo Y, Okayasu Y, et al. Association of D2-
40 and MMP-1 expression with cyst formation in lung metastatic lesions of cutaneous angiosarcoma on the scalp: immunohistochemical analysis of 23 autopsy cases. Human Pathology 2013;44:2751-9.

7. Maldonado L, Quadrelli S, Lyons G, Spina J, Venditti J, Chertcoff F. Neumotórax bilateral como complicación de metástasis pulmonar cavitaria de un Angiosarcoma. Medicina (Buenos Aires) 2014;74:227-8.

8. Jiménez D, Antaki J, Kamangar N. Metastatic pulmonary Angiosarcoma presenting with bilateral secondary spontaneous pneumothorax. Journal of Intensive Care Medicine 2017;32:292-6. 